This item was submitted to Loughborough's Institutional Repository (https://dspace.lboro.ac.uk/) by the author and is made available under the following Creative Commons Licence conditions.

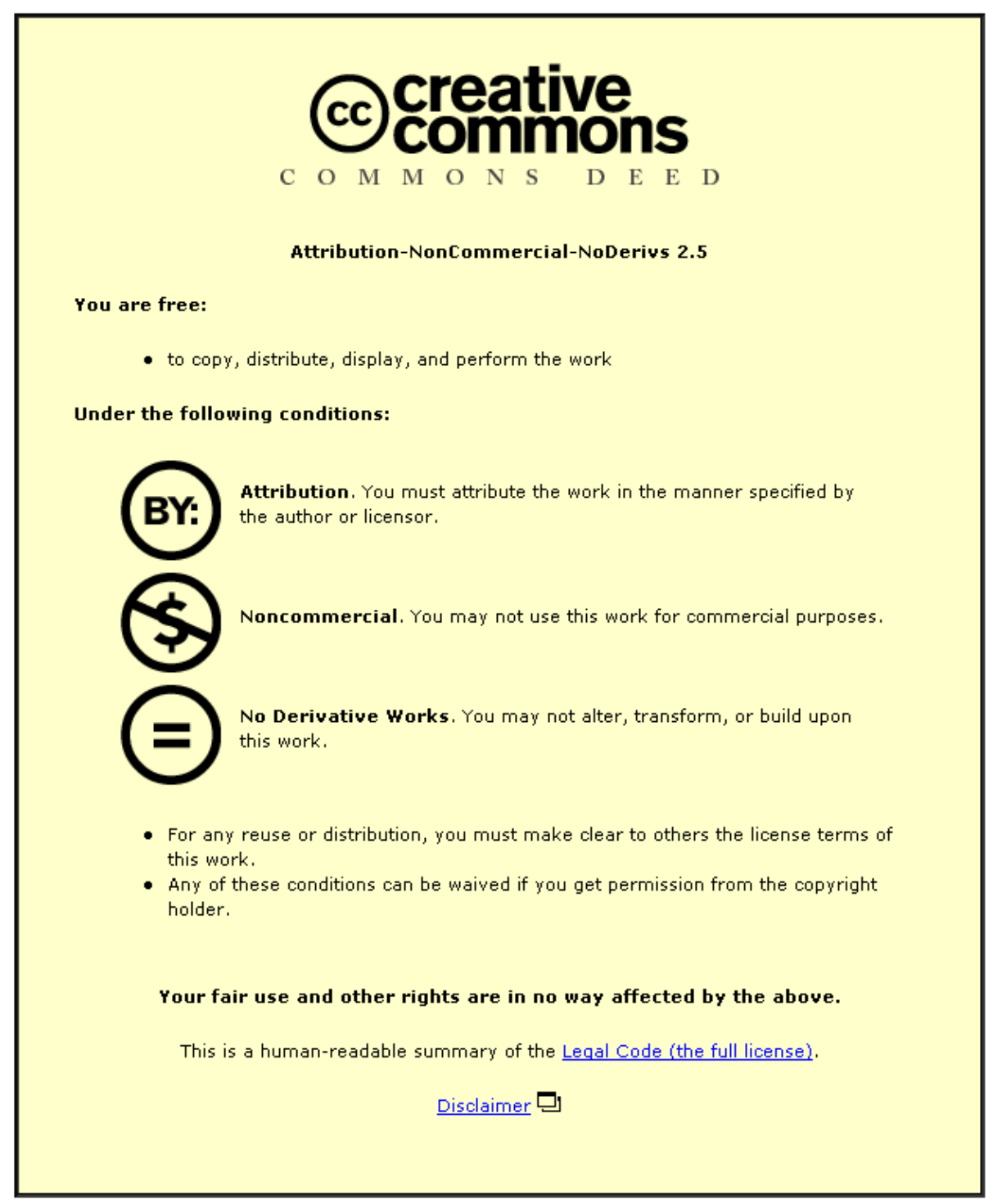

For the full text of this licence, please go to: http://creativecommons.org/licenses/by-nc-nd/2.5/ 
February 7, 2008

\title{
Generalized local interactions in 1D: solutions of quantum many-body systems describing distinguishable particles
}

\author{
Martin Hallnäs, Edwin Langmann and Cornelius Paufler \\ Mathematical Physics, Department of Physics, KTH-SCFAB, SE-106 91 \\ Stockholm, Sweden
}

\begin{abstract}
As is well-known, there exists a four parameter family of local interactions in 1D. We interpret these parameters as coupling constants of deltatype interactions which include different kinds of momentum dependent terms, and we determine all cases leading to many-body systems of distinguishable particles which are exactly solvable by the coordinate Bethe Ansatz. We find two such families of systems, one with two independent coupling constants deforming the well-known delta interaction model to non-identical particles, and the other with a particular one-parameter combination of the delta- and (so-called) delta-prime interaction. We also find that the model of non-identical particles gives rise to a somewhat unusual solution of the Yang-Baxter relations. For the other model we write down explicit formulas for all eigenfunctions.
\end{abstract}

\section{Introduction}

By general physical arguments one expects that, to understand the low-energy properties of a quantum system, one can ignore details of the short distance structure of the interactions and replace them by local interactions which are singular and non-trivial only at a point. The most prominent such local interaction is formally defined by a delta function potential and parameterized by one real parameter, but it is known since quite some time that, in one dimension (1D), the most general local interaction is characterized by four real parameters [1, 2]. While the earliest known examples of exactly solvable 1D quantum many-body systems with two-body interactions are defined with the delta function potential [3, 4, 5] (see also [6]), the full four parameter family of local interactions has received much 
less attention in this context until recently [7, 8, 9, 10, 11, 12, 13, 14, (see also Chapter 7 in [15]).

In this paper we consider the 1D quantum many-body systems with two-body interactions given by the general four parameter family of local interactions, and we determine all cases which are exactly solvable by the coordinate Bethe Ansatz even in the general case of distinguishable particles. (The same problem was also studied in [10, but our approach and results are different, as discussed in more detail below.) We find and solve two families of such models which provide interesting generalizations of previously known cases. We use the parameterization of the general local interaction proposed in [16], and we suggest a natural physical interpretation of these parameters as coupling constants of delta-type interactions which also include different kinds of momentum dependent terms. This allows us to write down formal Hamiltonians to define and interpret these models in a simple manner. While this interpretation is different from others in the literature [1, 17, the mathematically precise formulation of our models is the usual one in terms of boundary conditions. In the following two paragraphs we describe in more detail the exactly solvable models which we find.

The first family of models can be formally defined by the following Hamiltonian,

$$
\begin{aligned}
H^{(1)}= & -\sum_{j=1}^{N} \partial_{x_{j}}^{2}+\sum_{j<k}\left[2 c \delta\left(x_{j}-x_{k}\right)+2 \eta \mathrm{i}\left(\partial_{x_{j}}-\partial_{x_{k}}\right) \delta\left(x_{j}-x_{k}\right)\right. \\
& \left.+2 \eta \delta\left(x_{j}-x_{k}\right) \mathrm{i}\left(\partial_{x_{j}}-\partial_{x_{k}}\right)\right]
\end{aligned}
$$

which depends on two real coupling constants $c$ and $\eta$. For this and all other many-body models considered in this paper we assume that the particles move on the full real line, $x_{j} \in \mathbb{R}$ for $j=1,2, \ldots, N$, and we ignore bound states, which makes our solution complete only under certain restrictions on the coupling constants, such as $c>0 .{ }^{1}$ This model is a one-parameter extension of the famous 1D delta gas solved, in the boson case, by Lieb and Liniger [3], and in the general case of distinguishable particles by Yang using the coordinate Bethe Ansatz [5]. We interpret the extension as a particular momentum dependent interaction. ${ }^{2}$ We find that it is possible to generalize Yang's solution to the full model, even though it describes identical particles only in the Yang case $\eta=0$ (since the interaction terms with coupling $\eta$ are not invariant under particle exchanges).

A formal definition of the second model is

$$
H^{(2)}=-\sum_{j=1}^{N} \partial_{x_{j}}^{2}+\sum_{j<k}\left[2 c \delta\left(x_{j}-x_{k}\right)+\frac{2}{c}\left(\partial_{x_{j}}-\partial_{x_{k}}\right) \delta\left(x_{j}-x_{k}\right)\left(\partial_{x_{j}}-\partial_{x_{k}}\right)\right]
$$

\footnotetext{
${ }^{1}$ Bound states are of course interesting but beyond the scope of this work.

${ }^{2}$ Since $\hat{p}_{j} \equiv-\mathrm{i} \partial_{x_{j}}$ are the particle momentum operators.
} 
with only one real coupling parameter $c$. The first interaction term is identical to that of the 1D delta gas, but there is an additional momentum dependent interaction term with inverse coupling strength. As will be discussed below, this second interaction is identical with what is usually referred to as delta-prime interaction [2, 18]. This model describes identical particles, and it is thus possible to restrict it to bosons and fermions. It is interesting to note that, for fermions, the delta interaction is invisible (Pauli principle), and the model reduces to one first solved by Cheon and Shigehara [9], whereas for bosons the second interaction is invisible reducing it to the 1D Bose gas solved in [3] (see also [12]). We find that the explicit eigenfunctions of this model can be constructed in a remarkably simple manner by taking the well-known eigenfunctions of the boson delta gas restricted to the fundamental wedge $x_{1}<x_{2}<\ldots<x_{N}$ and extending it to all other wedges $x_{Q 1}<x_{Q 2}<\ldots<x_{Q N}, Q \in S_{N}$, using whatever particle statistics one considers. This generalizes the duality found in [9] to arbitrary exchange statistics.

We now recall some basic facts about general local interactions in 1D (see e.g. 16] for a more comprehensive discussion). The simplest example of a model with such interactions can be formally defined by a Hamiltonian

$$
H=-\partial_{x}^{2}+\hat{V}
$$

with $x \in \mathbb{R}$ the particle coordinate and $\hat{V}$ an interaction localized at $x=0$, i.e., the action on wave functions $\psi$ vanishes except at the origin. This implies that the Schrödinger equation determining the eigenstates $\psi$ of $H$ is trivial nearly everywhere, $\psi^{\prime \prime}+E \psi=0$ for $x \neq 0$, but the interaction results in non-trivial boundary conditions at the singular point $x=0$. Models of this type have been studied extensively using the theory of defect indices, from which it is known that the most general such interaction can be parameterized by four real parameters [1, 2]. The most prominent example is the delta function potential,

$$
\hat{V}=c \delta(x)
$$

parameterized by one real coupling constant $c$. Another well known special case is what often has been referred to as delta-prime interaction; see Section I.4 of 2]. Recently it was shown that the boundary conditions defining this interaction arise from the following momentum dependent potential,

$$
\hat{V}=4 \lambda \partial_{x} \delta(x) \partial_{x}
$$

with $\lambda$ a real coupling constant $\left[12 .{ }^{3}\right.$ In this paper we extend this point of view to the full four parameter family of local interactions, and we show that they arise

\footnotetext{
${ }^{3}$ This physical interpretation of the delta-prime interaction is similar to the one of Seba [18 but it does not require any renormalization of the coupling constant.
} 
from the following momentum dependent interaction,

$$
\hat{V}=c \delta(x)+4 \lambda \partial_{x} \delta(x) \partial_{x}+2(\gamma+\mathrm{i} \eta) \partial_{x} \delta(x)-2(\gamma-\mathrm{i} \eta) \delta(x) \partial_{x}
$$

where $c, \lambda, \gamma$ and $\eta$ are real coupling parameters. This interpretation is different from the standard one as a limit of conventional potentials which requires renormalizations of the coupling constants [17. However, it leads to a convenient parameterization $(c, \lambda, \gamma, \eta)$ of the local interactions which has a natural physical interpretation and which is without constraints. Moreover, as shown in Section 2 , by simple formal computations the interaction in (6) is turned into standard boundary conditions which provide a mathematically rigorous formulation of the model.

We can now give a more specific description of the many-body systems we consider. They are defined by the Hamiltonian

$$
H_{N}=-\sum_{j=1}^{N} \partial_{x_{j}}^{2}+\sum_{j<k} \hat{V}_{j k}
$$

with $x_{j} \in \mathbb{R}$ the particle coordinates and $\hat{V}_{j k}$ local two-body interactions obtained from the momentum dependent potential in (6) by replacing $x$ by the inter-particle distance $x_{j}-x_{k}$. In our discussion of special cases of this model we will use the notation $(c, \lambda, \gamma, \eta)$ introduced above, e.g., $(c, 0,0, \eta)$ refers to the model defined in (11). It is important to note that the general model $(c, \lambda, \gamma, \eta)$ describes identical particles only for $\eta=\gamma=0$ (since the interaction terms $\partial_{x} \delta(x) \pm \delta(x) \partial_{x}$ in (6) change sign under particle exchanges $\left.x \equiv x_{j}-x_{k} \rightarrow-x\right)$. Thus, besides the delta-interaction model $(c, 0,0,0)$, also the model $(0, \lambda, 0,0)$ is of special interest. However, different from the former, the latter is not exactly solvable in the general case of distinguishable particles [12]. Still, the restriction of this model to fermions is interesting since it provides the only non-trivial exactly solvable fermion model with local interactions in 1D (recall the the delta interaction is trivial for fermions; note that we only discuss models without internal degrees of freedom here). Moreover, it has a natural physical interpretation as the non-relativistic limit of the massive Thirring model [12]. Our results in this paper imply that the model in (2) is the only generalization of this fermion model to distinguishable particles which remains exactly solvable.

As already mentioned, the very same questions studied in this paper was also studied by Albeverio et. al. [10], ${ }^{4}$ but their results differ from ours. The reason for this is that in [10] it is assumed that the model describes identical particles so that the approach in [5] applies, and this restricts their analysis (implicitly) to the two parameter family $(c, \lambda, 0,0)$ of local interactions. Thus, while [10] also finds that

\footnotetext{
${ }^{4}$ We learnt about this work after finishing ours.
} 
the model in (2) is integrable, it concludes that the model (11) is integrable only for the previously known case $\eta=0 .{ }^{5}$ Indeed, one can check that the boundary conditions found to be integrable in [10], Eqs. (15) and (16), correspond to the cases $(c, 0,0,0)$ and $(q, 1 / q, 0,0)$ in our terminology. ${ }^{6}$ Thus, our main result in addition to [10] is the extension of Yang's approach [5] to models of non-identical particles and thereby giving the first conclusive answer to the question posed in the second paragraph above. Moreover, we also give a novel physical interpretation of these models.

The remainder of the paper is organized as follows. In Section 2 we discuss local interactions in $1 \mathrm{D}$, and in particular the relation of our interpretation to the standard one. In Section 3 we derive the consistency conditions on the coordinate Bethe Ansatz for the many-body systems with local interactions. Solving them we obtain the two families of systems defined in (11) and (2) (Section 3.2). Section 3.3 contains the explicit solution of the latter model. In Section 3.4 we construct the recursion relations for the coefficients arising in the coordinate Bethe Ansatz for the model in (11), which leads us to a somewhat unusual representation of the Yang-Baxter relations. We conclude with a few remarks on interesting related models not considered in this paper (Section 4). Some technical results related to our solution of the Yang-Baxter relation are deferred to two appendices.

\section{Local interactions in 1D}

Interactions localized at points in $1 \mathrm{D}$ have been studied extensively using the mathematical theory of defect indices; see [2] and references therein. From these studies it is well-known that the delta interaction is only one of many possible local interactions, and that a general such interaction can be characterized by four real coupling parameters. This can be formally understood as follows: for a 1D Hamiltonian $H=-\partial_{x}^{2}+\hat{V}$ with an interaction $\hat{V}$ localized at $x=0$, all eigenfunctions $\psi(x)$ should be smooth everywhere except at $x=0$, and $(H \psi)(x)=$ $-\psi^{\prime \prime}(x)$ for non-zero $x$. Requiring $H$ to be self-adjoint leads to the following consistency condition,

$$
\int_{|x|>0} \mathrm{~d} x\left(\overline{\phi^{\prime \prime}(x)} \psi(x)-\overline{\phi(x)} \psi^{\prime \prime}(x)\right)=0
$$

for arbitrary wave functions $\phi$ and $\psi$, or equivalently

$$
\left[\overline{\phi^{\prime}} \psi-\bar{\phi} \psi^{\prime}\right]_{x=0^{+}}=\left[\overline{\phi^{\prime}} \psi-\bar{\phi} \psi^{\prime}\right]_{x=-0^{+}}
$$

\footnotetext{
${ }^{5}$ In [10] a system is referred to as integrable if it is exactly solvable by the coordinate Bethe Ansatz.

${ }^{6}$ The third case obtained in [10] corresponds to the limiting case $\lim _{\eta \rightarrow \infty}\left(c \eta^{2}, 0, \eta, 0\right)$ in our terminology and thus violates the assumptions under which it was obtained.
} 
General boundary conditions are of the form

$$
\begin{aligned}
\psi^{\prime}\left(0^{+}\right) & =u_{11} \psi^{\prime}\left(-0^{+}\right)+u_{12} \psi\left(-0^{+}\right) \\
\psi\left(0^{+}\right) & =u_{21} \psi^{\prime}\left(-0^{+}\right)+u_{22} \psi\left(-0^{+}\right)
\end{aligned}
$$

(and similarly for $\phi$, of course) and are thus parameterized by four complex parameters $u_{j k}$ which, upon imposing (9), are reduced to two complex, or equivalently, four real parameters.

One prominent example of such consistent boundary conditions are

$$
\begin{aligned}
\psi\left(0^{+}\right) & =\psi\left(-0^{+}\right) \\
\psi^{\prime}\left(0^{+}\right)-\psi^{\prime}\left(-0^{+}\right) & =c \psi\left(0^{+}\right),
\end{aligned}
$$

which, as is well-known, corresponds to the delta interaction in (44). Another example is what is usually referred to as delta-prime interaction,

$$
\begin{array}{r}
\psi^{\prime}\left(0^{+}\right)=\psi^{\prime}\left(-0^{+}\right) \\
\psi\left(0^{+}\right)-\psi\left(-0^{+}\right)=4 \lambda \psi^{\prime}\left(0^{+}\right),
\end{array}
$$

and which corresponds to the momentum dependent interaction in (15).

As mentioned in the introduction, we use the following physical interpretation of the full four parameter family of local interactions,

$$
H=-\partial_{x}^{2}+\hat{V}, \quad \hat{V}=c \delta(x)+4 \lambda \partial_{x} \delta(x) \partial_{x}+2(\gamma+\mathrm{i} \eta) \partial_{x} \delta(x)-2(\gamma-\mathrm{i} \eta) \delta(x) \partial_{x},
$$

where $c, \lambda, \gamma$ and $\eta$ are real coupling constants. Once realized, this result is rather plausible: the operator $\hat{V}$ is obviously local and (formally) self-adjoint, and it contains four real parameters. Moreover, it is obviously the most general such interaction containing at most two derivatives, and it is plausible that higher derivatives than that cannot lead to consistent boundary conditions. It is also not so difficult to show that it indeed corresponds to the four parameter family of local interactions introduced in [1, 2]: formally, the eigenvalue equation of $H$ in (13) is equal to

$$
\begin{array}{r}
-\psi^{\prime \prime}(x)+c \delta(x) \psi(0)+4 \lambda \delta^{\prime}(x) \psi^{\prime}(0)+4 \lambda \delta(x) \psi^{\prime \prime}(0)+ \\
+2(\gamma+\mathrm{i} \eta) \delta^{\prime}(x) \psi(0)+2(\gamma+\mathrm{i} \eta) \delta(x) \psi^{\prime}(0)-2(\gamma-\mathrm{i} \eta) \delta(x) \psi^{\prime}(0)=E \psi(x)
\end{array}
$$

with $\psi$ the eigenfunction, $E$ the corresponding eigenvalue, and primes indicating derivatives. The crucial point is that, due to the singular interaction, the eigenfunctions are in general not continuous at the singular point $x=0$, and one therefore has to interpret the eigenfunction and its derivative at the singular point as the average of the left- and right limits,

$$
\begin{aligned}
\psi(0) & \equiv \frac{1}{2}\left[\psi\left(0^{+}\right)+\psi\left(-0^{+}\right)\right] \\
\psi^{\prime}(0) & \equiv \frac{1}{2}\left[\psi^{\prime}\left(0^{+}\right)+\psi^{\prime}\left(-0^{+}\right)\right] .
\end{aligned}
$$


Using that (14) can be turned into boundary conditions of the form (10) in the following way: integrating once from $x=-0^{+}$to $x=0^{+}$, and twice, first from $x=-0^{+}$to $x>0$ and then once more from $x=-0^{+}$to $x=0^{+}$gives

$$
\begin{aligned}
\psi^{\prime}\left(0^{+}\right)-\psi^{\prime}\left(-0^{+}\right) & =c \psi(0)-2(\gamma-\mathrm{i} \eta) \psi^{\prime}(0) \\
\psi\left(0^{+}\right)-\psi\left(-0^{+}\right) & =4 \lambda \psi^{\prime}(0)+2(\gamma+\mathrm{i} \eta) \psi(0) .
\end{aligned}
$$

It is interesting to note that this parameterization of boundary conditions is identical with the one proposed in [16], Eq. (2.1). Using (15) we can write these latter equations as in (10), with

$$
U \equiv\left(\begin{array}{ll}
u_{11} & u_{12} \\
u_{21} & u_{22}
\end{array}\right)=\left(U_{+}\right)^{-1} U_{-}, \quad U_{ \pm}=\left(\begin{array}{cc}
1 \pm(\gamma-\mathrm{i} \eta) & \mp c / 2 \\
\mp 2 \lambda & 1 \mp(\gamma+\mathrm{i} \eta)
\end{array}\right)
$$

where we introduce a convenient matrix notation. ${ }^{7}$ It is easily seen that the condition in (9) is equivalent to

$$
J \equiv\left(\begin{array}{cc}
0 & -1 \\
1 & 0
\end{array}\right)=U^{\dagger} J U
$$

which indeed is satisfied by the matrix $U$ in (17). We note that this physical interpretation provides a convenient parameterization of the full four parameter family of local interactions. In particular, it is without constraints, in this respect distinguishing it from others used in the literature, e.g. [1, 19]

$$
U=\mathrm{e}^{\mathrm{i} \chi}\left(\begin{array}{ll}
s & u \\
v & t
\end{array}\right), \quad \chi \in[0, \pi), \quad s, t, u, v \in \mathbb{R} \text { with } s t-u v=1 .
$$

Rephrasing our result in this latter parameterization is straightforward [16], but its physical interpretation would be less clear.

\section{Coordinate Bethe Ansatz}

In this section we consider the model of $N$ particles interacting via the full four parameter family of local two-body interactions. It is defined by the Hamiltonian $H_{N}$ in (7) with

$$
\begin{aligned}
\hat{V}_{j k}= & 2 c \delta\left(x_{j}-x_{k}\right)+2 \lambda\left(\partial_{x_{j}}-\partial_{x_{k}}\right) \delta\left(x_{j}-x_{k}\right)\left(\partial_{x_{j}}-\partial_{x_{k}}\right) \\
& +2(\gamma+\mathrm{i} \eta)\left(\partial_{x_{j}}-\partial_{x_{k}}\right)-2(\gamma-\mathrm{i} \eta) \delta\left(x_{j}-x_{k}\right)\left(\partial_{x_{j}}-\partial_{x_{k}}\right),
\end{aligned}
$$

\footnotetext{
${ }^{7}$ The special cases $\operatorname{det}\left(U_{+}\right)=0$ where $U$ above is undefined are discussed in [16]. We only mention that the case $\eta=\gamma=0$ and $\lambda=1 / c$ corresponds to separated boundary conditions, $\psi^{\prime}\left( \pm 0^{+}\right)=c \psi( \pm 0) / 2$.
} 
which is the obvious $N$ particle generalization of (6) $)$. Using $2 \partial_{x_{j}-x_{k}}=\partial_{x_{j}}-\partial_{x_{k}}$ it is straightforward to generalize the boundary conditions of the previous section to the $N$-particle case. We thus deduce that the eigenfunctions $\psi$ of $H_{N}$ are defined by the free Schrödinger equation

$$
\left(\sum_{j=1}^{N} \partial_{j}^{2}+E\right) \psi\left(x_{1}, \ldots, x_{N}\right)=0 \quad \text { for } \quad x_{j} \neq x_{k}
$$

and the following boundary conditions,

$$
\begin{array}{r}
\left(\partial_{x_{j}}-\partial_{x_{k}}\right)\left[\left.\psi\right|_{x_{j}=x_{k}+0^{+}}-\left.\psi\right|_{x_{j}=x_{k}-0^{+}}\right]=\left.2 c \psi\right|_{x_{j}=x_{k}} \\
-\left.2(\gamma-\mathrm{i} \eta)\left(\partial_{x_{j}}-\partial_{x_{k}}\right) \psi\right|_{x_{j}=x_{k}} \\
\left.\psi\right|_{x_{j}=x_{k}+0^{+}}-\left.\psi\right|_{x_{j}=x_{k}-0^{+}}=\left.2 \lambda\left(\partial_{x_{j}}-\partial_{x_{k}}\right) \psi\right|_{x_{j}=x_{k}}+\left.2(\gamma+\mathrm{i} \eta) \psi\right|_{x_{j}=x_{k}},
\end{array}
$$

where $j<k$ and where, similarly to the one particle case in the previous section, the eigenfunctions as well as their derivatives must be regularized at points of interaction $x_{j}=x_{k}$ as follows

$$
\begin{array}{r}
\left.\psi\right|_{x_{j}=x_{k}} \equiv \frac{1}{2}\left[\left.\psi\right|_{x_{j}=x_{k}+0^{+}}+\left.\psi\right|_{x_{j}=x_{k}-0^{+}}\right] \\
\left.\left(\partial_{x_{j}}-\partial_{x_{k}}\right) \psi\right|_{x_{j}=x_{k}} \equiv \frac{1}{2}\left(\partial_{x_{j}}-\partial_{x_{k}}\right)\left[\left.\psi\right|_{x_{j}=x_{k}+0^{+}}+\left.\psi\right|_{x_{j}=x_{k}-0^{+}}\right] .
\end{array}
$$

Equations (21) - (24) provide a mathematically rigorous formulation of our general model.

\subsection{Two particle case}

Before continuing our discussion of the full $N$ particle model we consider in some detail the two particle case, $N=2$, elucidating some of the properties that make this model rather special.

The most important such property is that the Hamiltonian $\mathrm{H}_{2}$ is invariant under permutations of the coordinates $x_{1}$ and $x_{2}$ only in the case $\gamma=\eta=0$, and that we therefore, in general, have a model of non-identical particles. To determine the implications this has on the eigenfunctions we start by considering the following Ansatz for the two particle scattering states,

$$
\phi= \begin{cases}\mathrm{e}^{\mathrm{i} k_{1} x_{1}+\mathrm{i} k_{2} x_{2}}+S_{R}^{+}\left(k_{1}-k_{2}\right) \mathrm{e}^{\mathrm{i} k_{2} x_{1}+\mathrm{i} k_{1} x_{2}}, & x_{1}<x_{2} \\ S_{T}^{+}\left(k_{1}-k_{2}\right) \mathrm{e}^{\mathrm{i} k_{1} x_{1}+\mathrm{i} k_{2} x_{2}}, & x_{2}<x_{1}\end{cases}
$$

which, upon substitution into boundary conditions (22), result in the following 
expressions for the two scattering amplitudes $S_{T}^{+}$and $S_{R}^{+}$,

$$
\begin{aligned}
S_{T}^{+}(u) & =\frac{\left(\gamma^{2}+\eta^{2}-2 \mathrm{i} \eta+c \lambda-1\right) u}{\mathrm{i} \lambda u^{2}-\left(\gamma^{2}+\eta^{2}+c \lambda+1\right) u-\mathrm{i} c} \\
S_{R}^{+}(u) & =\frac{\mathrm{i} \lambda u^{2}+2 \gamma u+\mathrm{i} c}{\mathrm{i} \lambda u^{2}-\left(\gamma^{2}+\eta^{2}+c \lambda+1\right) u-\mathrm{i} c},
\end{aligned}
$$

where we have introduced $u=k_{1}-k_{2}$. For future convenience we introduce also the scattering amplitudes $S_{R}^{-}$and $S_{T}^{-}$obtained from $S_{R}^{+}$and $S_{T}^{+}$by reverting the sign of $\gamma$ and $\eta$,

$$
S_{R}^{-}=\left.S_{R}^{+}\right|_{\gamma \rightarrow-\gamma, \eta \rightarrow-\eta}, \quad S_{T}^{-}=\left.S_{T}^{+}\right|_{\gamma \rightarrow-\gamma, \eta \rightarrow-\eta} .
$$

From this Ansatz we construct a second set of eigenfunctions of $H_{2}$ by noting that the Hamiltonian $H_{2}$, while not invariant under the exchange of the coordinates $x_{1}$ and $x_{2}$, is invariant under the simultaneous exchange of the coordinates $x_{1}$ and $x_{2}$ and replacement of $\gamma$ and $\eta$ by $-\gamma$ and $-\eta$ respectively. This implies that also $\left.\phi\right|_{x_{1} \leftrightarrow x_{2}, \gamma \rightarrow-\gamma, \eta \rightarrow-\eta}$ is an eigenfunction of $H_{2}$, or equivalently, that $\left.\phi\right|_{x_{1} \leftrightarrow x_{2}}$ is an eigenfunction of $\left.H_{2}\right|_{\gamma \rightarrow-\gamma, \eta \rightarrow-\eta}$. Note that $\phi$ and $\left.\phi\right|_{x_{1} \leftrightarrow x_{2}, \gamma \rightarrow-\gamma, \eta \rightarrow-\eta}$ are linearly independent. Since the potential $\hat{V}_{12}=0$ unless $x_{1}=x_{2}$, every eigenfunction $\psi$ of $H_{2}$ obeys the free Schrödinger equation $\left(\partial_{1}^{2}+\partial_{2}^{2}+E\right) \psi\left(x_{1}, x_{2}\right)=0$ in all regions without coinciding coordinates. Thus, in these regions they are linear combinations of the plane waves $\mathrm{e}^{\mathrm{i} k_{1} x_{1}+\mathrm{i} k_{2} x_{2}}$ and $\mathrm{e}^{\mathrm{i} k_{2} x_{1}+\mathrm{i} k_{1} x_{2}}$. This implies that $\phi$ and $\left.\phi\right|_{x_{1} \leftrightarrow x_{2}, \gamma \rightarrow-\gamma, \eta \rightarrow-\eta}$ provide, in the absence of bound states, a complete set of eigenfunctions of $\mathrm{H}_{2}$.

\subsection{Consistency conditions}

We now consider, for arbitrary $N$, the model defined by $H_{N}$ in (17) and (20). In particular, we determine for which values of the coupling constants $(c, \lambda, \gamma, \eta)$ its eigenfunctions $\psi(x)$ can be obtained by the coordinate Bethe Ansatz

$$
\psi(x)=\sum_{P \in S_{N}} A_{P}(Q) \mathrm{e}^{\mathrm{i} k_{P} \cdot x_{Q}}
$$

in the wedge

$$
\Delta_{Q}: \quad x_{Q(1)}<x_{Q(2)}<\ldots<x_{Q(N)}
$$

with $x=\left(x_{1}, \ldots, x_{N}\right)$ and $k_{P} \cdot x_{Q}=\sum_{j=1}^{N} k_{P(j)} x_{Q(j)}$, for all $Q \in S_{N}$ [5]. The corresponding eigenvalue is obviously $E=\sum_{j=1}^{N} k_{j}^{2}$. We recall that the validity of the Bethe Ansatz amounts to the model being quantum integrable in the sense that the most that can happen in any two-body scattering process is an exchange of particle momenta. 
To take the boundary conditions (22) into account we consider the boundary $x_{j}=x_{k}$ for fixed $j$ and $k$ such that $j<k$. The last requirement is important since the particles in general are non-identical, as previously discussed. Furthermore, let $Q$ be an element in $S_{N}$ such that $x_{Q(i)} \equiv x_{j}$ and $x_{Q(i+1)} \equiv x_{k}$ for some fixed $i$, implying that $x_{j}=x_{k}-0^{+}$is contained in the wedge $\Delta_{Q}$ and $x_{j}=x_{k}+0^{+}$in $\Delta_{Q T_{i}}$, where $T_{i} \in S_{N}$ is the transposition interchanging $i$ and $i+1$. From this we deduce that the boundary conditions imply the following relations between the coefficients $A_{P}(Q)$ of the coordinate Bethe Ansatz (28),

$$
\begin{array}{r}
\mathrm{i}\left(k_{P(i)}-k_{P(i+1)}\right)\left[A_{P T_{i}}\left(Q T_{i}\right)-A_{P}\left(Q T_{i}\right)-A_{P}(Q)+A_{P T_{i}}(Q)\right]=c\left[A_{P}(Q)\right. \\
\left.+A_{P T_{i}}(Q)+A_{P}\left(Q T_{i}\right)+A_{P T_{i}}\left(Q T_{i}\right)\right]-(\mathrm{i} \gamma+\eta)\left[A_{P T_{i}}\left(Q T_{i}\right)-A_{P}\left(Q T_{i}\right)\right. \\
\left.+A_{P}(Q)-A_{P T_{i}}(Q)\right] \\
\begin{array}{r}
A_{P}\left(Q T_{i}\right)+A_{P T_{i}}\left(Q T_{i}\right)-A_{P}(Q)-A_{P T_{i}}(Q)=\mathrm{i} \lambda\left(k_{P(i)}-k_{P(i+1)}\right)\left[A_{P T_{i}}\left(Q T_{i}\right)\right. \\
\left.-A_{P}\left(Q T_{i}\right)+A_{P}(Q)-A_{P T_{i}}(Q)\right]+(\gamma+\mathrm{i} \eta)\left[A_{P}(Q)+A_{P T_{i}}(Q)\right. \\
\left.+A_{P}\left(Q T_{i}\right)+A_{P T_{i}}\left(Q T_{i}\right)\right] .
\end{array}
\end{array}
$$

These relations constitute a linear, homogeneous system of $2(N-1) N !^{2}$ equations for the $N !^{2}$ unknowns $A_{P}(Q)$, and the Bethe Ansatz is consistent if and only if this over-determined system of equations has $N$ ! independent solutions where the $A_{P}(I)$ can be chosen arbitrarily. In the following discussion we will show that this is the case in the two special cases corresponding to the models defined by (II) and (2). To do this we will not attempt to solve these rather complicated system of equations by brute-force, but rather use a somewhat indirect approach which essentially amounts to reducing the $N$-particle case to a sequence of two particle problems.

Lemma 3.1. Let $Q \in S_{N}$ and $i \in\{1, \ldots, N\}$. If $Q(i)<Q(i+1)$ and the coordinate Bethe Ansatz (28) is consistent, then

$$
\begin{gathered}
A_{P T_{i}}(Q)=S_{R}^{+}\left(k_{P(i)}-k_{P(i+1)}\right) A_{P}(Q)+S_{T}^{-}\left(k_{P(i)}-k_{P(i+1)}\right) A_{P}\left(Q T_{i}\right) \\
A_{P T_{i}}\left(Q T_{i}\right)=S_{R}^{-}\left(k_{P(i)}-k_{P(i+1)}\right) A_{P}\left(Q T_{i}\right)+S_{T}^{+}\left(k_{P(i)}-k_{P(i+1)}\right) A_{P}(Q),
\end{gathered}
$$

where $T_{i}$ is the transposition interchanging $i$ and $i+1$.

Proof. We first consider the case $N=2$. Let $\psi$ be an arbitrary eigenfunction of $H_{2}$. Recall that the eigenfunctions $\phi$ and $\left.\phi\right|_{x_{1} \leftrightarrow x_{2}, \gamma \rightarrow-\gamma, \eta \rightarrow-\eta}$ of $H_{2}$ constitute, in the absence of bound states, a complete basis for the eigenspace of $H_{2}$. It follows that $\psi$ is a linear combination,

$$
\begin{aligned}
\psi & =a_{1} \phi+\left.a_{2} \phi\right|_{x_{1} \leftrightarrow x_{2}, \gamma, \eta \rightarrow-\gamma,-\eta} \\
& =\left\{\begin{array}{ll}
a_{1} \mathrm{e}^{\mathrm{i} k_{1} x_{1}+\mathrm{i} k_{2} x_{2}}+f_{1}\left(k_{1}-k_{2}\right) \mathrm{e}^{\mathrm{i} k_{2} x_{1}+\mathrm{i} k_{1} x_{2}}, & x_{1}<x_{2} \\
f_{2}\left(k_{1}-k_{2}\right) \mathrm{e}^{\mathrm{i} k_{2} x_{2}+\mathrm{i} k_{1} x_{1}}+a_{2} \mathrm{e}^{\mathrm{i} k_{1} x_{2}+\mathrm{i} k_{2} x_{1}}, & x_{2}<x_{1}
\end{array},\right.
\end{aligned}
$$


for some complex constants $a_{1}$ and $a_{2}$; we have introduced the functions

$$
\begin{gathered}
f_{1}\left(k_{1}-k_{2}\right)=S_{R}^{+}\left(k_{1}-k_{2}\right) a_{1}+S_{T}^{-}\left(k_{1}-k_{2}\right) a_{2} \\
f_{2}\left(k_{1}-k_{2}\right)=S_{R}^{-}\left(k_{1}-k_{2}\right) a_{2}+S_{T}^{+}\left(k_{1}-k_{2}\right) a_{1} .
\end{gathered}
$$

Relabeling the constants $a_{1}$ and $a_{2}$ as well as the functions $f_{1}$ and $f_{2}$ as follows, $a_{1}=A_{I}(I), a_{2}=A_{I}\left(T_{1}\right), A_{T_{1}}(I)=f_{1}$ and $A_{T_{1}}\left(T_{1}\right)=f_{2}$, we arrive at the twoparticle Bethe Ansatz with coefficients $A_{P}(Q)$ given by

$$
\begin{array}{r}
A_{T_{1}}(I)=S_{R}^{+}\left(k_{1}-k_{2}\right) A_{I}(I)+S_{T}^{-}\left(k_{1}-k_{2}\right) A_{I}\left(T_{1}\right) \\
A_{T_{1}}\left(T_{1}\right)=S_{R}^{-}\left(k_{1}-k_{2}\right) A_{I}\left(T_{1}\right)+S_{T}^{+}\left(k_{1}-k_{2}\right) A_{I}(I) .
\end{array}
$$

To extend this result to arbitrary values of $N$ we observe that the boundary conditions (22) become identical with the boundary conditions for the case $N=2$ if we substitute $x_{j} \rightarrow x_{1}$ and $x_{k} \rightarrow x_{2}$, for all $j<k$. For each $P \in S_{N}$, let $\Delta_{Q}$ be a wedge such that $x_{Q i} \equiv x_{j}$ and $x_{Q(i+1)} \equiv x_{k}$. From the equivalence of boundary conditions, in the sense stated above, follows that the relations between the coefficients $A_{P}(Q), A_{P}\left(Q T_{i}\right), A_{P T_{i}}(Q)$ and $A_{P T_{i}}\left(Q T_{i}\right)$ are obtained from (34) by the substitutions $x_{1} \rightarrow x_{Q(i)}$ and $x_{2} \rightarrow x_{Q(i+1)}$ as well as $k_{1} \rightarrow k_{P(i)}$ and $k_{2} \rightarrow k_{P(i+1)}$. This yields the relations in (31).

It is important to note that there is a possible inconsistency in the coordinate Bethe Ansatz arising from the fact that the representation of an element in $S_{N}$ is not unique. However, any two representations can be converted into each other by using the defining relations of $S_{N}$,

$$
\begin{array}{r}
T_{i} T_{i}=1, \quad T_{i} T_{i+1} T_{i}=T_{i+1} T_{i} T_{i+1} \\
T_{i} T_{j}=T_{j} T_{i} \quad \text { for }|i-j|>1 .
\end{array}
$$

Thus no inconsistency can arise provided that

$$
\begin{array}{r}
A_{P T_{i} T_{i}}(Q)=A_{P}(Q), \quad A_{P T_{i} T_{i+1} T_{i}}(Q)=A_{P T_{i+1} T_{i} T_{i+1}}(Q) \\
A_{P T_{i} T_{j}}(Q)=A_{P T_{j} T_{i}}(Q) \text { for }|i-j|>1
\end{array}
$$

for all $P, Q \in S_{N}$.

To determine when these conditions are valid we follow the approach of A. B. and Al. B. Zamolodchikov [20] and make use of the algebraic structure which has come to be known as the Zamolodchikov algebra. We start by briefly recalling the construction of the Zamolodchikov algebra: to each particle of type $A$ and with momenta $k$ the symbol $A(k)$ is associated, and the two particle scattering theory is encoded in the commutation relation

$$
A\left(k_{1}\right) B\left(k_{2}\right)=S_{R}^{A B}\left(k_{12}\right) A\left(k_{2}\right) B\left(k_{1}\right)+S_{T}^{A B}\left(k_{12}\right) B\left(k_{2}\right) A\left(k_{1}\right),
$$


where $k_{12}=k_{1}-k_{2}$ and $S_{R}^{A B}$ and $S_{T}^{A B}$ are the two particle scattering amplitudes. The full $N$ particle scattering theory is then obtained by factorizing each scattering event into a product of two particle events. As observed in [20, identifying each product $A\left(k_{1}\right) B\left(k_{2}\right) C\left(k_{3}\right) \ldots$ with a particular coefficient $A_{P}(Q)$ of the coordinate Bethe Ansatz the consistency conditions (36) are equivalent to requiring the Zamolodchikov algebra to be consistent as well as associative. In our particular case, since the particles are distinguished only by their relative ordering, it is sufficient to consider the case of three particles. Therefore, let the product $A\left(k_{1}\right) B\left(k_{2}\right) C\left(k_{3}\right)$ correspond to a particular coefficient $A_{P}(Q)$, for fixed $P, Q \in S_{3}$. A straightforward but somewhat tedious computation then shows that the Zamolodchikov algebra, in our case, is consistent as well as associative if and only if the two-particle scattering amplitudes $S_{R}^{ \pm}$and $S_{T}^{ \pm}$obey the following so called Factorization equations,

$$
\begin{aligned}
& S_{R}^{+}(u) S_{R}^{+}(-u)+S_{T}^{-}(u) S_{T}^{+}(-u)=1 \\
& S_{R}^{-}(u) S_{R}^{-}(-u)+S_{T}^{+}(u) S_{T}^{-}(-u)=1 \\
& S_{R}^{+}(u) S_{T}^{-}(-u)+S_{T}^{-}(u) S_{R}^{-}(-u)=0 \\
& S_{R}^{-}(u) S_{T}^{+}(-u)+S_{T}^{+}(u) S_{R}^{+}(-u)=0 \\
& S_{R}^{-}(v) S_{R}^{+}(u+v) S_{R}^{-}(u)=S_{R}^{+}(u) S_{R}^{-}(u+v) S_{R}^{+}(v) \\
& S_{R}^{+}(v) S_{T}^{+}(u+v) S_{T}^{-}(u)=S_{T}^{+}(u) S_{T}^{-}(u+v) S_{R}^{+}(v) \\
& S_{R}^{-}(v) S_{T}^{-}(u+v) S_{T}^{+}(u)=S_{T}^{-}(u) S_{T}^{+}(u+v) S_{R}^{-}(v) \\
& S_{R}^{+}(v) S_{R}^{+}(u+v) S_{T}^{-}(u)+S_{T}^{-}(v) S_{R}^{+}(u+v) S_{R}^{-}(u)=S_{R}^{+}(u) S_{T}^{-}(u+v) S_{R}^{+}(v) \\
& S_{R}^{-}(v) S_{R}^{+}(u+v) S_{T}^{+}(u)+S_{T}^{+}(v) S_{R}^{+}(u+v) S_{R}^{+}(u)=S_{R}^{+}(u) S_{T}^{+}(u+v) S_{R}^{+}(v) \\
& S_{R}^{-}(v) S_{R}^{-}(u+v) S_{T}^{+}(u)+S_{T}^{+}(v) S_{R}^{-}(u+v) S_{R}^{+}(u)=S_{R}^{-}(u) S_{T}^{+}(u+v) S_{R}^{-}(v) \\
& S_{R}^{+}(v) S_{R}^{-}(u+v) S_{T}^{-}(u)+S_{T}^{-}(v) S_{R}^{+}(u+v) S_{R}^{-}(u)=S_{R}^{-}(u) S_{T}^{-}(u+v) S_{R}^{+}(v) \\
& S_{R}^{+}(v) S_{R}^{-}(u+v) S_{T}^{-}(u)+S_{T}^{-}(v) S_{R}^{-}(u+v) S_{R}^{-}(u)=S_{R}^{-}(u) S_{T}^{-}(u+v) S_{R}^{-}(v) \\
& S_{R}^{-}(v) S_{R}^{+}(u+v) S_{T}^{+}(u)+S_{T}^{+}(v) S_{R}^{-}(u+v) S_{R}^{+}(u)=S_{R}^{+}(u) S_{T}^{+}(u+v) S_{R}^{-}(v)
\end{aligned}
$$

for all real $u$ and $v$.

Upon substituting the two-particle scattering amplitudes (26) and (27) into the Factorization equations above a straightforward but somewhat tedious calculation shows that (38)-(41) as well as (43)-(44) are fulfilled for all values of $(c, \lambda, \gamma, \eta)$, while (42) and (45)-(50) holds true if and only if

$$
\begin{aligned}
\gamma\left[\lambda\left(3 c+\lambda\left(u^{2}+u v+v^{2}\right)\right)+4 \gamma^{2}\right] & =0 \\
{\left[-1+c \lambda+\gamma^{2}+\eta(\eta-2 \mathrm{i})\right]\left[\lambda\left(3 c+\lambda\left(u^{2}+u v+v^{2}\right)\right)+4 \gamma^{2}\right] } & =0 \\
{\left[-1+c \lambda+\gamma^{2}+\eta(\eta-2 \mathrm{i})\right]\left[\lambda\left(3 c+\lambda\left(u^{2}+u v+v^{2}\right)\right)+4 \gamma^{2}\right] } & =0
\end{aligned}
$$

for all real $u$ and $v$. Using the fact that the first condition requires $\gamma=0$ and that the last two are related by complex conjugation, we can reduce them to the 
rather simple form

$$
\begin{aligned}
\gamma & =0 \\
\lambda[-1+c \lambda+\eta(\eta-2 \mathrm{i})] & =0,
\end{aligned}
$$

which obviously has two families of solutions, each of which defines systems exactly solvable by the coordinate Bethe Ansatz. We thus arrive at the main result of this paper.

Theorem 3.2. The coordinate Bethe Ansatz (28) for the eigenfunctions of the Hamiltonian $H_{N}$ is consistent if and only if

$$
\lambda=\gamma=0
$$

or

$$
\lambda=1 / c \quad \text { and } \quad \gamma=\eta=0 .
$$

Note that these two sets of conditions on the coupling constants $(c, \lambda, \gamma, \eta)$ correspond to the two Hamiltonians defined in (11) and (2). It is interesting to note that, in both cases, $S_{R}^{ \pm}$and $S_{T}^{ \pm}$only have a single pole, and $S_{R}^{+}=S_{R}^{-}$even in the first case.

\subsection{Explicit results for the case $(c, 1 / c, 0,0)$}

This particular case correspond to the Hamiltonian $H^{(2)}$ in Eq. (21). We note that this Hamiltonian is invariant under permutations of the coordinates $x_{j}$, and one can therefore assume a particular exchange statistics, which determines the eigenfunction in all wedges once it is known in one of them. Furthermore, using the fact that $\left.S_{T}^{+}\right|_{\lambda=1 / c, \gamma=\eta=0}=\left.S_{T}^{-}\right|_{\lambda=1 / c, \gamma=\eta=0}=0$ and $\left.S_{R}^{+}\right|_{\lambda=1 / c, \gamma=\eta=0}=\left.S_{R}^{-}\right|_{\lambda=1 / c, \gamma=\eta=0}$, the relations in (31) reduce to the rather simple form

$$
\begin{aligned}
A_{P T_{i}}(Q) & =\left.S_{R}^{+}\right|_{\lambda=1 / c, \gamma=\eta=0}\left(k_{P(i)}-k_{P(i+1)}\right) A_{P}(Q) \\
& =\frac{\mathrm{i}\left(k_{P(i)}-k_{P(i+1)}\right)^{2}+\mathrm{i} c^{2}}{\mathrm{i}\left(k_{P(i)}-k_{P(i+1)}\right)^{2}+2\left(k_{P(i)}-k_{P(i+1)}\right)-\mathrm{i} c^{2}} A_{P}(Q) \\
& =\frac{\mathrm{i}\left(k_{P(i)}-k_{P(i+1)}\right)-c}{\mathrm{i}\left(k_{P(i)}-k_{P(i+1)}\right)+c} A_{P}(Q)
\end{aligned}
$$

for all $Q \in S_{N}$. This implies that

$$
A_{P T_{i}}=Y_{i}\left(k_{P(i)}-k_{P(i+1)}\right) A_{P},
$$

where we have introduced the function

$$
Y_{i}(u)=\frac{\mathrm{i} u+c}{\mathrm{i} u-c},
$$


and interpret $A_{P}$ as a vector with $N$ ! elements $A_{P}(Q)$. It is important to note that the functions $Y_{i}$, for arbitrary exchange statistics, are identical to the corresponding functions appearing when restricting the delta-interaction model to bosons (see e.g. 21]). The eigenfunctions of the latter model are well-known (see e.g. Section I.1 in [22])

$$
\psi=C\left[\prod_{N \geq j>k \geq 1}\left(\partial_{x_{j}}-\partial_{x_{k}}+c\right)\right] \operatorname{det}_{1 \leq m, n \leq N}\left[\exp \left(\mathrm{i} k_{m} x_{n}\right)\right], \quad x_{1}<x_{2}<\ldots<x_{N}
$$

where $C$ is a normalization constant. We conclude that this formula also gives the eigenfunctions of the Hamiltonian in (2) in the identity wedge $\Delta_{I}$, for arbitrary exchange statistics (characterized by some Young tableaux). Using standard arguments from group theory (see e.g. [23]), these eigenfunctions can be straightforwardly extended to all other wedges.

\subsection{Recursion relations for the coefficients $A_{P}$}

In order to provide a machinery for computing the eigenfunctions explicitly we now proceed to derive a set of recursion relations for the coefficients $A_{P}$ of the coordinate Bethe Ansatz. Our starting point is the following fact: defining

$$
(\hat{R})_{Q, Q^{\prime}}=\delta_{Q^{\prime}, Q R}
$$

one can write

$$
A_{P}(Q R)=\sum_{Q^{\prime} \in W_{N}}(\hat{R})_{Q, Q^{\prime}} A_{P}\left(Q^{\prime}\right)=\left(\hat{R} A_{P}\right)(Q)
$$

where the first equality is a trivial consequence of the definition, and in the second we interpret $(\hat{R})_{Q, Q^{\prime}}$ as elements of an $n \times n$ matrix $\hat{R}$ with $n=N$ ! the $\operatorname{rank}\left|S_{N}\right|$ of $S_{N}$. These matrices obviously define a representation $R \rightarrow \hat{R}$ of $S_{N}$ acting on the coefficients $A_{P}(Q)$. It is worth noting that this is identical with the so called (right) regular representation of $S_{N}$. Using this fact we can rewrite the two relations in (31) as follows,

$$
\begin{array}{r}
A_{P T_{i}}(Q)=\left[S_{R}^{+}\left(k_{P(i)}-k_{P(i+1)}\right)+S_{T}^{-}\left(k_{P(i)}-k_{P(i+1)}\right) \hat{T}_{i}\right] A_{P}(Q) \\
A_{P T_{i}}\left(Q T_{i}\right)=\left[S_{R}^{-}\left(k_{P(i)}-k_{P(i+1)}\right)+S_{T}^{+}\left(k_{P(i)}-k_{P(i+1)}\right) \hat{T}_{i}\right] A_{P}\left(Q T_{i}\right),
\end{array}
$$

where $Q$ is required to fulfill the condition $Q(i)<Q(i+1)$. To organize the elements of the vector $A_{P}$ we have to introduce an ordering on the set of permutations. We find it convenient to use the following 
Definition 3.3. Associate to any two permutations $Q, Q^{\prime} \in S_{N}$ the sequence $a_{i}:=Q(N-i+1)-Q^{\prime}(N-i+1)$, where $i=1,2, \ldots, N$. If the first non-zero number in the sequence $\left\{a_{i}\right\}$ is positive, $Q$ is said to be larger than $Q^{\prime}$, denoted as $Q>Q^{\prime}$.

The idea for the remainder is the following: order the coefficients $A_{P}(Q)$ according to the ordering just defined (largest permutation first) into the vector $A_{P}$, and use the recursion relations in (61) for the individual coefficients $A_{P}(Q)$ to write

$$
A_{P T_{i}}=\mathbf{Y}_{i}\left(k_{P(i)}-k_{P(i+1)}\right) A_{P},
$$

where we have introduced the matrix

$$
\mathbf{Y}_{i}(u)=\mathbf{S}_{R}^{i}(u)+\mathbf{S}_{T}^{i}(u) \hat{T}_{i}
$$

in which $\mathbf{S}_{R}^{i}$ and $\mathbf{S}_{T}^{i}$ are diagonal $N ! \times N !$ matrices with entries $S_{R}^{ \pm}$and $S_{T}^{ \pm}$ respectively. To determine the distribution of the scattering amplitudes $S_{R}^{ \pm}$and $S_{T}^{ \pm}$among the diagonal elements of the matrices $\mathbf{S}_{R}^{i}$ and $\mathbf{S}_{T}^{i}$ we start by deducing a natural decomposition of an arbitrary permutation into a product of elementary transpositions $T_{i}$. This decomposition will provide us with enough information to determine the structure of $\mathbf{S}_{R}^{i}$ and $\mathbf{S}_{T}^{i}$ explicitly.

We pause to introduce some notation and conventions to be used in the remainder of the discussion. By abuse of notation, we identify a permutation $Q \in S_{N}$ with $Q^{\prime} \in S_{N+n}, n \geq 0$, if

$$
\begin{array}{r}
Q(i)=Q^{\prime}(i) \text { for all } i=1,2, \ldots, N \\
Q^{\prime}(i)=i \text { for all } i=N+1, N+2, \ldots, n
\end{array}
$$

E.g., $(231) \in S_{3}$ and $(23145) \in S_{5}$ will be identified. ${ }^{8}$ Furthermore, we denote as $Q_{k}^{m}$ the $k$ th permutation of $S_{m}$, ordered according to the ordering defined in Definition 3.3. E.g. for $S_{3}$ this implies that $Q_{1}^{3}=(123), Q_{2}^{3}=(213), Q_{3}^{3}=(132)$, $Q_{4}^{3}=(312), Q_{5}^{3}=(231)$ and $Q_{6}^{3}=(321)$.

Using the fact that every integer $j$ such that $1 \leq j \leq m$ ! can be uniquely written in the form $j=n(m-1) !+k$, where $0 \leq n \leq m-1$ and $1 \leq k \leq(m-1)$ !, we prove

Lemma 3.4. Let $k, m$ and $n$ be integers such that $m>1,0 \leq n \leq m-1$ and $1 \leq k \leq(m-1)$ !. Then

$$
Q_{n(m-1) !+k}^{m}=\prod_{i=m-n}^{\stackrel{m-1}{\longrightarrow}} T_{i} Q_{k}^{m-1},
$$

\footnotetext{
${ }^{8}$ We recall that a permutation $Q=(i j k)$ is defined such that $Q(1)=i, Q(2)=j$ and $Q(3)=k$, etc.
} 
where

$$
\stackrel{k}{\longrightarrow} T_{i=j}=\left\{\begin{array}{ll}
T_{j} T_{j+1} \ldots T_{k}, & j \leq k \\
0 & \text { otherwise }
\end{array} .\right.
$$

Proof. It clearly follows from Definition 3.3 and the fact $\left|S_{m}\right|=m$ ! that $Q_{n(m-1) !+1}^{m}$ is obtained by cyclically permuting the last $m-n$ elements of the identity permutation in $S_{m}$ such that $Q_{n(m-1) !+1}^{m}(m)=m-n$. Decomposing this cyclic permutation into a product of elementary transpositions $T_{i}$, we obtain

$$
Q_{n(m-1) !+1}^{m}=T_{m-n} \ldots T_{m-1} .
$$

We conclude the proof of the Lemma by observing that

$$
Q_{n(m-1) !+k}^{m}=Q_{n(m-1) !+1}^{m} Q_{k}^{m-1} .
$$

Corollary 3.5. Let $k, m$ and $n$ be integers such that $m>1,0 \leq n \leq m$ and $1 \leq k \leq m$ !. Then

$$
\begin{gathered}
Q_{n m !+k}^{m+1}(m)>Q_{n m !+k}^{m+1}(m+1), \quad 1 \leq k \leq n(m-1) ! \\
Q_{n m !+k}^{m+1}(m)<Q_{n m !+k}^{m+1}(m+1), \quad n(m-1) !<k \leq m ! .
\end{gathered}
$$

Proof. From Lemma 3.4 clearly follows that $Q_{n m !+1}^{m+1}(m+1)=m+1-n$, and consequently that

$$
\begin{gathered}
Q_{n m !+1}^{m+1}(j)<Q_{n m !+1}^{m+1}(m+1), \quad 1 \leq j \leq m-n \\
Q_{n m !+1}^{m+1}(j)>Q_{n m !+1}^{m+1}(m+1), \quad m-n<j \leq m .
\end{gathered}
$$

In other words, for each fixed $n$ there exists $n$ distinct integers $l \leq m+1$ such that $l>Q_{n m !+1}^{m+1}(m+1)$. This, together with the facts that $Q_{n m !+k}^{m+1}=Q_{n m !+1}^{m+1} Q_{k}^{m}$ and that $Q_{k}^{m}(m)=m$, for all $k \leq(m-1)$ !, imply the statement.

Note that Lemma 3.4 provides a recursive procedure for decomposing an arbitrary permutation into a product of elementary transpositions $T_{i}$.

Using Lemma 3.4 and Corollary 3.5 we now determine the structure of the matrices $\mathbf{S}_{R}^{i}$ and $\mathbf{S}_{T}^{i}$. Corollary 3.5] and the recursion relations (61) together imply that

$$
\left(\mathbf{S}_{R}^{i}\right)_{j j}= \begin{cases}S_{R}^{-} & 1 \leq j-n i ! \leq n(i-1) ! \\ S_{R}^{+} & n(i-1) !<j-n i ! \leq i !\end{cases}
$$

and

$$
\left(\mathbf{S}_{T}^{i}\right)_{j j}=\left\{\begin{array}{ll}
S_{T}^{+} & 1 \leq j-n i ! \leq n(i-1) ! \\
S_{T}^{-} & n(i-1) !<j-n i ! \leq i !
\end{array},\right.
$$


where $n$ is required to fulfill the relation $j=n i !+k$, for some $1 \leq k \leq i$ !. This determines only the first $(i+1)$ ! diagonal elements of the $N ! \times N$ ! matrices $\mathbf{S}_{R}^{i}$ and $\mathbf{S}_{T}^{i}$. However, it immediately follows from Lemma 3.4 that this structure is periodic with period $(i+1)$ !, i.e.,

$$
\begin{gathered}
\left(\mathbf{S}_{R}^{i}\right)_{j+(i+1) !, j+(i+1) !}=\left(\mathbf{S}_{R}^{i}\right)_{j j} \\
\left(\mathbf{S}_{T}^{i}\right)_{j+(i+1) !, j+(i+1) !}=\left(\mathbf{S}_{T}^{i}\right)_{j j},
\end{gathered}
$$

thus determining all $N !^{2}$ elements of $\mathbf{S}_{R}^{i}$ and $\mathbf{S}_{T}^{i}$. We recall that for our case,

$$
S_{T}^{ \pm}(u)=\frac{\left(-\eta^{2} \pm 2 \mathrm{i} \eta+1\right) u}{\left(\eta^{2}+1\right) u+\mathrm{i} c}, \quad S_{R}^{ \pm}(u)=\frac{-\mathrm{i} c}{\left(\eta^{2}+1\right) u+\mathrm{i} c}
$$

but we give the construction for the more general case where $S_{R}^{ \pm}$are different since we hope that this will be useful for other models.

In deriving the recursion relations (62) it is important to note that there is a possible inconsistency in the coordinate Bethe Ansatz, arising from the fact that the elementary transpositions $T_{i}$ obey the defining relations (35) of the permutation group $S_{N}$, as discussed in Section 3.2. Thus the recursion relations (62) are consistent if and only if

$$
\begin{aligned}
A_{P T_{i} T_{i}}= & A_{P}, \quad A_{P T_{i} T_{i+1} T_{i}}=A_{P T_{i+1} T_{i} T_{i+1}} \\
& A_{P T_{i} T_{j}}=A_{P T_{j} T_{i}} \text { for }|i-j|>1
\end{aligned}
$$

for all $P \in S_{N}$. Using the recursion relations (62) one find that that these latter conditions holds true if and only if the following so called Yang-Baxter relations are fulfilled,

$$
\begin{array}{r}
\mathbf{Y}_{i}(-u) \mathbf{Y}_{i}(u)=I, \quad \mathbf{Y}_{i}(v) \mathbf{Y}_{i+1}(u+v) \mathbf{Y}_{i}(u)=\mathbf{Y}_{i+1}(u) \mathbf{Y}_{i}(u+v) \mathbf{Y}_{i+1}(v) \\
\mathbf{Y}_{i}(u) \mathbf{Y}_{j}(v)=\mathbf{Y}_{j}(v) \mathbf{Y}_{i}(u) \text { for }|i-j|>1
\end{array}
$$

for all real $u$ and $v$. However, since we already in Section 3.2 determined for which values of the coupling parameters $(c, \lambda, \gamma, \eta)$ the relations (75) holds true we can, in view of Theorem 3.2 , immediately conclude that we have proven

Proposition 3.6. If and only if the coupling parameters $(c, \lambda, \gamma, \eta)$ satisfy the conditions in (53) or (54), then the Yang-Baxter relations (76) are fulfilled, and

$$
A_{P}=\mathcal{Y}_{P}(k) A_{I}
$$

where $\mathcal{Y}_{P}(k)$ is a product of the matrices $\mathbf{Y}_{i}\left(k_{P(i)}-k_{P(i+1)}\right)$ obtained by repeatedly using (62). 
Remark 3.7. In the special case $\gamma=\eta=0$ the Hamiltonian $H_{N}$ is invariant under permutations of the coordinates, and in consequence the scattering amplitude $\left.S_{R}^{+}\right|_{\gamma=\eta=0}=\left.S_{R}^{-}\right|_{\gamma=\eta=0}=: S_{R}$ and similarly $\left.S_{T}^{+}\right|_{\gamma=\eta=0}=\left.S_{T}^{-}\right|_{\gamma=\eta=0}=: S_{T}$. A particularly interesting special case is the delta interaction model $\lambda=\gamma=\eta=0$, in which case the matrices $\mathbf{Y}_{i}$ take the well known form

$$
\left.\mathbf{Y}_{i}(u)\right|_{\lambda=\gamma=\eta=0}=\frac{\mathrm{i} u \hat{T}_{i}+c \hat{I}}{\mathrm{i} u-c},
$$

originally introduced by Yang [5].

To illustrate the general discussion of this section we provide in Appendix A a more explicit account of the three particle case $N=3$. In Appendix B we outline a simple, direct proof of the Yang-Baxter equations for the cases stated in Proposition 3.6.

\section{Concluding remarks}

In the introduction we argued that the Hamiltonian $H_{N}$ defined in (7) and (20) is the $N$ particle generalization of the most general Hamiltonian $H=-\partial_{x}^{2}+\hat{V}$ with local interaction $\hat{V}$. However, not all $N$-body Hamiltonians with local two-body interactions can be obtained in this way. A particular example of such a model is the quantum version of the derivative nonlinear Schrödinger equation 24] defined by the Hamiltonian

$$
H_{N}=-\sum_{j=1}^{N} \partial_{x_{j}}^{2}+2 \tilde{\eta} \sum_{j<k} \delta\left(x_{j}-x_{k}\right) \mathrm{i}\left(\partial_{x_{j}}+\partial_{x_{k}}\right),
$$

where $\tilde{\eta}$ is a real coupling parameter. This model does not fall in the class studied in this paper since the interaction depends also on the sum of the momenta of the particles involved in the interaction, and it is therefore not Galilean invariant. However, this Hamiltonian is nevertheless interesting. It describes identical particles, and it was shown by Gutkin [7] that it is exactly solvable by the coordinate Bethe Ansatz only in the case of bosons or fermions.

All possible local two-body interactions are formally defined by the 2-body Hamiltonian $H_{2}=-\partial_{x_{1}}^{2}-\partial_{x_{2}}^{2}+\hat{W}_{12}$, where $\left(\hat{W}_{12} \psi\right)\left(x_{1}, x_{2}\right)=0$ for two-particle wave functions $\psi$ except in regions of coinciding coordinates, $x_{1}=x_{2}$. We believe that there is a nine parameter family of such two-body interactions which one should be able to find using general methods discussed in [2, 15]. It would be interesting to know if there are additional such distinguishable particle models exactly solvable by the coordinate Bethe Ansatz. 
As discussed in [16] Remark 2.4, there is a one parameter family of local interactions which are unitarily equivalent to the non-interacting case. This suggests that there should be also a one parameter extension of local interactions which are unitarily equivalent to the delta-interaction, and this might provide a simple explanation of our results. At closer inspection we found that this is indeed the case: if and only if $\psi(x)$ obeys the boundary conditions in (16) defining the interaction $(c, 0, \eta, 0)$, then

$$
\tilde{\psi}(x)=\mathrm{e}^{-\mathrm{i} \alpha \Theta(x)} \psi(x), \quad \mathrm{e}^{\mathrm{i} \alpha} \equiv \frac{1+\mathrm{i} \eta}{1-\mathrm{i} \eta}
$$

with the Heaviside function $\Theta$ obeys the boundary conditions defining the interaction $\left(c /\left(1+\eta^{2}\right), 0,0,0\right)$. In a similar manner, the model formally defined in (11) is unitarily equivalent to the delta-gas with coupling $\tilde{c}=c /\left(1+\eta^{2}\right)$, and the unitary operator $U$ intertwining the two models is given by

$$
(U \psi)\left(x_{1}, \ldots, x_{N}\right)=\mathrm{e}^{-\mathrm{i} \alpha \sum_{j<k} \Theta\left(x_{j}-x_{k}\right)} \psi\left(x_{1}, \ldots, x_{N}\right)
$$

with $\alpha$ as above. ${ }^{9}$ Moreover, it is interesting to note that (74) can be written as

$$
S_{T}^{ \pm}(u)=\mathrm{e}^{ \pm \mathrm{i} \alpha} b(u), \quad S_{R}^{ \pm}(u)=a(u)
$$

where $Y_{i}(u)=a(u)+b(u) T_{i}$ gives the rational solution of the Yang-Baxter relations, suggesting that our solution $\mathbf{Y}_{i}(u)$ of the Yang-Baxter relations is unitary equivalent to the latter. We should mention that this very argument was used already in 11] to deduce the integrability of the model in (11) ${ }^{10}$ However, while this is a simple alternative argument showing integrability of the model in (11), only our argument allows to conclusively determine all integrable cases.

To conclude, in this paper we found an example of a 1D quantum many-body system of non-identical particles which is integrable, and it led us to an interesting class of solutions of the Yang-Baxter relations. While the solution which we found is closely related to the well-known rational one, there might be others which are truly different from known ones, and our results provide a convenient starting point for finding them. It thus is tempting to speculate that our model is only a first example in a novel interesting class of integrable systems.

\section{Acknowledgements}

We would like to thank Harald Grosse for asking us the question answered in this paper, as well as for his encouragement along the many phases of this project. We

\footnotetext{
${ }^{9}$ We stress that this unitary equivalence cannot be understood on the level of formal Hamiltonians since our local interactions include non-trivial regularizations.

${ }^{10}$ We thank P. Kurasov for pointing this out to us.
} 
would also like to thank P. Kulish for several helpful remarks. C.P. was supported by Deutsche Forschungsgemeinschaft under the Emmy-Noether Programme. E.L. was supported in part by the Swedish Science Research Council (VR) and the Göran Gustafsson Foundation.

\section{Appendix A. Recursion relations for the three particle case}

To illustrate the abstract discussion of Section 3.4 we here provide explicit formulas for the three particle case. We start by noting that the vector $A_{P}$, in the ordering defined in Section 3.3, has the following structure,

$$
A_{P}^{t}=\left(A_{P}(123), A_{P}(213), A_{P}(132), A_{P}(312), A_{P}(231), A_{P}(321)\right),
$$

where $A_{P}^{t}$ denotes the transpose of $A_{P}$. Straightforward computations (or alternatively Lemma 3.4 show that we can write this using the elementary transpositions $T_{1}$ and $T_{2}$ as follows,

$$
A_{P}^{t}=\left(A_{P}(I), A_{P}\left(T_{1}\right), A_{P}\left(T_{2}\right), A_{P}\left(T_{1} T_{2}\right), A_{P}\left(T_{2} T_{1}\right), A_{P}\left(T_{1} T_{2} T_{1}\right)\right)
$$

Directly applying the recursion relations (61) we find that the matrices $S_{R}^{1}$ and $S_{T}^{1}$, in the particular ordering we have chosen, take the following form,

$$
\mathbf{S}_{R}^{1}=\left(\begin{array}{cccccc}
S_{R}^{+} & 0 & 0 & 0 & 0 & 0 \\
0 & S_{R}^{-} & 0 & 0 & 0 & 0 \\
0 & 0 & S_{R}^{+} & 0 & 0 & 0 \\
0 & 0 & 0 & S_{R}^{-} & 0 & 0 \\
0 & 0 & 0 & 0 & S_{R}^{+} & 0 \\
0 & 0 & 0 & 0 & 0 & S_{R}^{-}
\end{array}\right), \quad \mathbf{S}_{T}^{1}=\left(\begin{array}{cccccc}
S_{T}^{-} & 0 & 0 & 0 & 0 & 0 \\
0 & S_{T}^{+} & 0 & 0 & 0 & 0 \\
0 & 0 & S_{T}^{-} & 0 & 0 & 0 \\
0 & 0 & 0 & S_{T}^{+} & 0 & 0 \\
0 & 0 & 0 & 0 & S_{T}^{-} & 0 \\
0 & 0 & 0 & 0 & 0 & S_{T}^{+}
\end{array}\right) .
$$

Using $T_{1} T_{2} T_{1}=T_{2} T_{1} T_{2}$ and (61) we deduce that

$$
\mathbf{S}_{R}^{2}=\left(\begin{array}{cccccc}
S_{R}^{+} & 0 & 0 & 0 & 0 & 0 \\
0 & S_{R}^{+} & 0 & 0 & 0 & 0 \\
0 & 0 & S_{R}^{-} & 0 & 0 & 0 \\
0 & 0 & 0 & S_{R}^{+} & 0 & 0 \\
0 & 0 & 0 & 0 & S_{R}^{-} & 0 \\
0 & 0 & 0 & 0 & 0 & S_{R}^{-}
\end{array}\right), \quad \mathbf{S}_{T}^{2}=\left(\begin{array}{cccccc}
S_{T}^{-} & 0 & 0 & 0 & 0 & 0 \\
0 & S_{T}^{-} & 0 & 0 & 0 & 0 \\
0 & 0 & S_{T}^{+} & 0 & 0 & 0 \\
0 & 0 & 0 & S_{T}^{-} & 0 & 0 \\
0 & 0 & 0 & 0 & S_{T}^{+} & 0 \\
0 & 0 & 0 & 0 & 0 & S_{T}^{+}
\end{array}\right) .
$$

It is straightforward to verify that this structure is indeed reproduced also by the general discussion presented in Section 3.3, in particular Eqs. (71)-(173). To explicitly construct the matrices $\mathbf{Y}_{i}$, for $i=1,2$, and thereby also the recursion 
relations (62), there remains only to determine the explicit form of $\hat{T}_{1}$ and $\hat{T}_{2}$. Using (59) we find that

$$
\hat{T}_{1}=\left(\begin{array}{cccccc}
0 & 1 & 0 & 0 & 0 & 0 \\
1 & 0 & 0 & 0 & 0 & 0 \\
0 & 0 & 0 & 1 & 0 & 0 \\
0 & 0 & 1 & 0 & 0 & 0 \\
0 & 0 & 0 & 0 & 0 & 1 \\
0 & 0 & 0 & 0 & 1 & 0
\end{array}\right), \quad \hat{T}_{2}=\left(\begin{array}{llllll}
0 & 0 & 1 & 0 & 0 & 0 \\
0 & 0 & 0 & 0 & 1 & 0 \\
1 & 0 & 0 & 0 & 0 & 0 \\
0 & 0 & 0 & 0 & 0 & 1 \\
0 & 1 & 0 & 0 & 0 & 0 \\
0 & 0 & 0 & 1 & 0 & 0
\end{array}\right) .
$$

Inserting the above matrices into the consistency conditions (76) a straightforward but somewhat tedious calculation shows that they are equivalent to Factorization equations (38)-(50). This reflects the fact that, for this model, it is sufficient to consider the three-particle case in order to find those cases exactly solvable by the coordinate Bethe Ansatz.

\section{Appendix B. Direct proof of Yang-Baxter equa- tions}

In this Appendix we outline an alternative, direct proof of the validity of the Yang-Baxter relations in (76) for the operators $\mathbf{Y}_{i}(u)$ defined in Eqs. (63) and (26) -(27) and the cases stated in Theorem 3.2.

We first note that the formulas given in Appendix A allow a simple verification of these relations in the simplest non-trivial case $N=3 .{ }^{11}$ The general result, for arbitrary $N$, then follows from the following

Lemma B.1. If the operators $\mathbf{Y}_{i}(u)$ satisfy the Yang-Baxter relations for $N=3$ they satisfy them for any $N>2$.

This is true since all identities in (76) can be brought to a block diagonal form, where the relations in each block are unitarily equivalent to the corresponding ones for the case $N=3$.

To be more specific, we consider first the second identity in (76)), involving three matrices $\mathbf{Y}$ on each side, for some fixed $N>2$ and $i<N$. It follows from (63) that $\mathbf{Y}_{i}(u)$ acting on $A_{P}$ mixes only elements $A_{P}(Q)$ and $A_{P}\left(Q T_{i}\right)$ (since $\mathbf{S}_{R, T}^{i}$ are diagonal matrices). Thus, for any $Q \in S_{N}$, both sides of the second set of identities in (76) mix only the elements $A_{P}(Q), A_{P}\left(Q T_{i}\right), A_{P}\left(Q T_{i+1}\right)$, $A_{P}\left(Q T_{i+1} T_{i}\right)$ and $A_{P}\left(Q T_{i} T_{i+1}\right)$, i.e., it is possible to reorder the elements of the vector $A_{P}$ so that one can group them into blocks of six elements such that the

\footnotetext{
${ }^{11}$ The verification can easily be performed by using a symbolic programming language like MAPLE or MATHEMATICA.
} 
identities decompose into blocks of $6 \times 6$ matrices. Moreover, let $Q^{\prime}$ be the largest element, when using the ordering defined in Definition 3.3. in the set $\left\{Q, Q T_{i}, Q T_{i+1}, Q T_{i+1} T_{i}, Q T_{i} T_{i+1}, Q T_{i+1} T_{i}\right\}$, then

$$
Q^{\prime}>Q^{\prime} T_{i}>Q^{\prime} T_{i+1}>Q^{\prime} T_{i+1} T_{i}>Q^{\prime} T_{i} T_{i+1}>Q^{\prime} T_{i} T_{i+1} T_{i}
$$

This implies that the matrices $\mathbf{Y}_{i}(u)$ and $\mathbf{Y}_{i+1}(u)$ restricted to the vector

$$
\left(A_{P}\left(Q^{\prime}\right), A_{P}\left(Q^{\prime} T_{i}\right), A_{P}\left(Q^{\prime} T_{i+1}\right), A_{P}\left(Q^{\prime} T_{i+1} T_{i}\right), A_{P}\left(Q^{\prime} T_{i} T_{i+1}\right), A_{P}\left(Q^{\prime} T_{i} T_{i+1} T_{i}\right)\right)^{t}
$$

are identical with the $6 \times 6$ matrices $\mathbf{Y}_{1}(u)$ and $\mathbf{Y}_{2}(u)$ for $N=3$ respectively (these latter matrices are explicitly given in Appendix A). This proves the lemma for the second set of identities in (176). The verification of the first and last set of identities follows from similar arguments (they reduce to blocks of $2 \times 2$ and $4 \times 4$ matrix identities which are easy to verify).

\section{References}

[1] P. Šeba, Regularized Potentials in Nonrelativistic Quantum Mechanics. I. The one-dimensional case, Czech. J. Phys. B 36, 455 (1986).

[2] S. Albeverio, F. Gesztesy, R. Høegh-Krohn, and H. Holden, Solvable Models in Quantum Mechanics, Springer-Verlag, Heidelberg (1988).

[3] E. H. Lieb and W. Liniger, Exact analysis of an interacting Bose gas. I. The general solution and the ground state, Phys. Rev. 130, 1605 (1963).

[4] J. B. McGuire, Study of Exactly Soluble One-Dimensional N-Body Problems, J. Math. Phys. 5, 622 (1964).

[5] C. N. Yang, Some exact results for the many-body problem in one dimension with repulsive delta-function interaction, Phys. Rev. Lett. 19, 1312 (1967).

[6] M. Gaudin, La fonction d'onde de Bethe, Masson, Paris (1983).

[7] E. Gutkin, Bethe Ansatz and the Generalized Yang-Baxter Equations, Annals. Phys. 176, 22 (1987).

[8] F. A. B. Coutinho, Y. Nogami and Lauro Tomio, Many-body system with a four-parameter family of point interactions in one dimension, J. Phys. A: Math. Gen. 32, 4931 (1999).

[9] T. Cheon and T. Shigehara, Fermion-Boson Duality of One-Dimensional Quantum Particles with Generalized Contact Interactions, Phys. Rev. Lett. 82, 2536 (1999). 
[10] S. Albeverio, S-M. Fei and P. Kurasov, On Integrability of Many-Body Problems with Point Interactions, Operator Theory: Advances and Applications, 132, 67 (2002).

[11] S. Albeverio, S-M. Fei and P. Kurasov, Gauge fields, point interactions and few-body problems in one dimension, Rept. Math. Phys. 53, 363 (2004).

[12] H. Grosse, E. Langmann and C. Paufler, Exact solution of a 1D many-body system with momentum dependent interactions, J. Phys. A 37, 4579 (2004).

[13] V. Caudrelier, M. Mintchev and E. Ragoucy, The quantum non-linear Schrödinger model with point-like defect, J. Phys. A 37, L367 (2004).

[14] M. Hallnäs and E. Langmann, Exact solutions of two complementary 1D quantum many-body systems on the half-line, math-ph/0404023.

[15] S. Albeverio and P. Kurasov, Singular Perturbations of Differential Operators, Cambridge Univ. Press, Cambridge (2000).

[16] P. Exner and H. Grosse, Some properties of the one-dimensional generalized point interactions (a torso), math-ph/9910029.

[17] T. Cheon and T. Shigehara, Realizing discontinuous wave functions with renormalized short-range potentials, Phys. Lett. A 243, 111 (1998).

[18] P. Šeba, Some Remarks on the $\delta^{\prime}$-Interaction in One Dimension, Rep. Math. Phys. 24, 111 (1986).

[19] S. Albeverio, L. Dąbrowski and P. Kurasov, Symmetries of Schrödinger operators with point interactions, Lett. Math. Phys. 45, 33 (1998).

[20] A. B. Zamolodchikov and Al. B. Zamolodchikov, Factorized S-matrices in Two Dimensions as the Exact Solutions of Certain Relativistic Quantum Field Theory Models, Annals Phys. 120, 253 (1979).

[21] Z. Chang, Quantum groups and quantum symmetry, Phys. Rept. 262, 137 (1995).

[22] V. E. Korepin and N. M. Bogoliubov and A. G. Izergin, Quantum Inverse Scattering and Correlation Functions, Cambridge university press, Cambridge (1993).

[23] H. Weyl, Theory of Groups and Quantum Mechanics, Dover Publications (1984).

[24] D. J. Kaup and A. C. Newell, An exact solution for a derivative nonlinear Schrödinger equation, J. Math. Phys. 19, 798 (1978). 